\title{
Asymptotic Estimates of Fundamental Solutions of Third order Nonlinear Difference Equation
}

\section{Ananthan ${ }^{1}$ and S. Kandasamy ${ }^{2}$}

${ }^{1}$ Department of Mathematics, Aarupadai veedu Institute of Technology,

Vinayaka Missions University, Paiyanoor, Kancheepuram-603104, Tamilnadu, India.

2Department of Mathematics, Mahendra Engineering College (Autonomous),

Namakkal-637 503, Tamilnadu, India.

ABSTRACT: We find asymptotic estimates of fundamental solutions of third order Difference equation $y(n+3)-\left(2+e^{-n-2}\right) y(n+2)-\left(1+\frac{1}{n^{2}+1}\right) y(n+1)+2 y(n)=0$

KEYWORDS: Difference equation; Oscillation and Non oscillation; asymptotic properties; polynomial.

\section{INTRODUCTION}

The asymptotic behavior of third order difference equation play a central role in many branches of pure and applied mathematics such as continued fractions, special functions, orthogonal polynomials and combinatorics. In this paper we will utilize the special characteristics of third order equation to obtain a deeper understanding of the asymptotic of their solution. The difference equation of the form

$y(n+3)-\left(2+e^{-n-2}\right) y(n+2)-\left(1+\frac{1}{n^{2}+1}\right) y(n+$

$$
1+2 y n=0
$$

The characteristic equation is given by $\lambda^{3}-$ $2 \lambda^{2}-\lambda+2=0$ with roots $\lambda_{1}=2, \lambda_{2}=1, \lambda_{3}=-1$. Notice that $p_{1}(n)=-e^{-n-2}, p_{2}(n)=-\frac{1}{n^{2}+1}, p_{3}(n)=0 \quad$ all satisfy below condition (2). Hence from the corollary (1) applies to produce solutions $y_{1}(n), y_{2}(n)$ and $y_{3}(n)$ defined as follows

$y_{1}(n)=[1+o(1)] 2^{n}, \quad y_{2}(n)=1+o(1), \quad y_{3}(n)=[1+$ $o(1)](-1)^{n}$.

Corollary (1) is due to Evgrafov. It says that for each characteristic root of polynomial (2), at least one solution behaves in formula $y_{i}(n)=[1+o(1)] \lambda_{i}^{n}$, provided that the rate of convergence of the coefficients is not too slow.

Corollary: 1

Suppose that the polynomial

$$
\begin{aligned}
& p(\lambda)=\lambda^{k}+a_{1} \lambda^{k-1}+\cdots \ldots \ldots \ldots \ldots \ldots+a_{k} \\
& \text { Have distinct roots } \lambda_{1}, \lambda_{2}, \ldots \ldots \ldots \ldots \ldots \lambda_{k} \\
& \text { and that }
\end{aligned}
$$

$$
\sum_{n=1}^{\infty}\left|p_{i}(n)\right| \prec \infty \quad \text { for } 1 \leq i \leq k \text {. }
$$

Then equation $y(n+k)+\left(a_{1}+p_{1}(n)\right) y(n+k-1)+\ldots \ldots \ldots \ldots+\left(a_{k}+p_{k}(n)\right) y(n)=0$ (A)

has k solutions

$$
y_{1}(n), y_{2}(n), \ldots \ldots \ldots \ldots . . . y_{k}(n)
$$

with

$$
y_{i}(n)=[1+o(1)] \lambda_{i}^{n}
$$

Proof:

First we put into the form of a k-dimensional system

$(n+1)=[A+B(n)] z(n)$

$$
y(n+k)+\left(a_{1}+p_{1}(n)\right) y(n+k-1)+\ldots \ldots \ldots \ldots+\left(a_{k}+p_{k}(n)\right) y(n)=0
$$
where

$$
\mathrm{A}=\left(\begin{array}{ccccc}
0 & 1 & \cdot & \cdot & 0 \\
0 & 9 & \cdot & 1 & 0 \\
\cdot & \cdot & \cdot & \cdot & \cdot \\
\cdot & \cdot & \cdot & \cdot & \cdot \\
-a_{k} & -a_{k-1} & \cdot & \cdot & -a_{1}
\end{array}\right)
$$

${ }^{*}$ Corresponding Author: kandasamythamarai@gmail.com

Received: 12.10 .2017

Accepted: 10.11 .2017

Published on: 12.12 .2017

\section{V.Ananthan and S.Kandasamy}




$$
\begin{gathered}
B(n)=\left(\begin{array}{ccccc}
0 & 0 & . & . & 0 \\
0 & 0 & . & 1 & 0 \\
\cdot & . & . & . & . . \\
\cdot & . . & . & \cdot & . \\
\cdot & \cdot & . & . & . \\
-p_{k}(n) & -p_{k-1}(n) & . & . & -p_{1}(n)
\end{array}\right) \\
z(n)=(y(n), y(n+1), \ldots . . y(n+k-1))^{T}
\end{gathered}
$$

Notice that polynomial (2) is the characteristic polynomial of the matrix A. Furthermore, for each eigen value $\lambda_{i}$ there corresponds the eigen vectors $\xi=\left(1, \lambda_{i}^{1}, \lambda_{i}^{2}, \ldots \ldots \ldots . \lambda_{i}^{k-1}\right)^{T}$. In addition, the matrix $B(n)$ satisfies condition

$\sum_{n=n_{0}\left|\lambda_{i}(n)\right|}^{\infty}\|B(n)\| \prec \infty$

(6)

Hence one may apply theorem (1.1) to conclude that there are k solutions

$Z_{1}(n), Z_{2}(n), \ldots \ldots \ldots \cdot Z_{k}(n)$ of (5) such that, for $1 \leq i \leq k$.

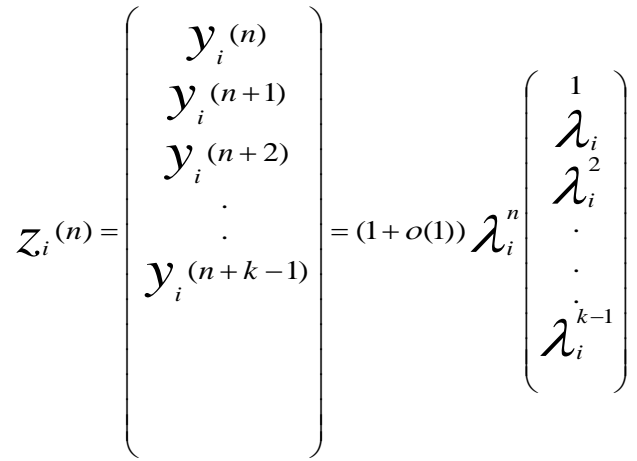

Hence $y_{i}(n)=[1+o(1)] \lambda_{i}^{n}$

\section{Theorem 1.1}

Suppose that the matrix A has k linearly independent eigen values $\xi_{1}, \xi_{2}, \ldots . \xi_{k}$ and $\mathrm{k}$ corresponding eigen vectors $\lambda_{1}, \lambda_{2}, \ldots \ldots \lambda_{k}$.If condition $\sum_{n=n_{0}}^{\infty} \frac{1}{\left|\lambda_{i}(n)\right|}\|B(n)\|<\infty$ holds for $B(n)$, then system of equation

$y(n+1)=[A+B(n)] y(n)$

has solutions

$y_{i}(n), 1 \leq i \leq k$, such that $y_{i}(n)=\left[\xi_{i}+o(1)\right] \lambda_{i}^{n}$
Proof:

In order to be able to apply theorem 1.2 we need to diagonalize the matrix A. This may be accomplished by letting

$y=T z$

In equation (6) where $T=\left(\xi_{1}, \xi_{2}, \ldots \ldots \ldots \xi_{k}\right)$

That is the $\mathrm{i}$-th column of $\mathrm{T}$ is $\xi$. Then we obtain

$T z(n+1)=[A+B(n)] T z(n)$

(or)

$z(n+1)=[D+B(n)] z(n)$

Where $D=T^{-1} A T=\operatorname{diag}\left(\lambda_{1}, \lambda_{2}, \ldots \ldots \ldots \lambda_{k}\right)$ and $\overline{B(n)}$

$=T^{-1} B(n) T$. It is easy to see that $B(n)$ satisfies conditions (A). Now formula (9) follows by applying theorem1.1.

\section{Theorem 1.2}

Suppose that condition

Condition

$L=\left\{\begin{array}{c}\prod_{r=0}^{n}\left|\frac{\lambda_{i}(r)}{\lambda_{j}(r)}\right| \rightarrow+\infty \\ \text { and } \prod_{r=n_{1}}^{n_{2}}\left|\frac{\lambda_{i}(r)}{\lambda_{j}(r)}\right| \geq \mu>0, \text { for all } 0 \leq n_{1} \leq n_{2}, \\ \text { or } \prod_{r=n_{1}}^{n_{2}}\left|\frac{\lambda_{i}(r)}{\lambda_{j}(r)}\right| \leq k, \text { for all } 0 \leq n_{1} \leq n_{2}\end{array}\right.$

Holds and for each $i, 1 \leq i \leq k, \sum_{n=n_{0}}^{\infty} \frac{1}{\left|\lambda_{i}(n)\right|}\|B(n)\|<$ $\infty$.

(A)

Then the equation $y(n+1)=(D(n)+B(n)) y(n)$ has a fundamental set of $\mathrm{k}$ solutions $y_{i}(n)$ such that $y_{i}(n)=\left(e_{i}+o(1)\right) \prod_{r=n_{0}}^{n-1} \lambda_{i}(n)$

Where $e_{i}$ is the standard unit vector in $R^{+}$where its components are all zero, except that the $\mathrm{i}$-th component is 1.

Theorem 1.3

Suppose that the polynomial (2) has a k-fold root of 1 and that

$\sum_{n=1}^{\infty} n^{k-1}\left|p_{i}(n)\right|<\infty$ for $1 \leq i \leq k$

Then

$y(n+k)+\left(a_{1}+p_{1}(n)\right) y(n+k-1)+\cdots$

$\left(a_{k}+p_{k}(n)\right) y(n)=0$

Have k solutions $y_{1}(n), y_{2}(n), \ldots \ldots \ldots y_{k}(n)$ with

$y_{i}(n)=n^{i-1}(1+o(1)), n \rightarrow \infty$

Solution:

We remark here that the actual result of Coffman is stronger than the statement of theorem 1.3. Indeed, he proved that

$\Delta^{m} y_{i}(n)=\left\{\begin{array}{c}\left(\begin{array}{c}n \\ i-m\end{array}\right)+o\left(\begin{array}{c}i-m \\ n\end{array}\right) \text { for } 1 \leq m \leq i \\ o\left(\begin{array}{c}i-m \\ n\end{array}\right) \text { for } i \leq m \leq k-1\end{array}\right.$

The curious reader might wonder coffman's theorem applies if the polynomial (2) has a k-fold root not equal to 1 . Luckly by a very simple trick, one is able to do exactly that. Assume that the characteristic equation (2) has a $\mathrm{k}$-fold root $\mu \neq 1$. Then polynomial (2) may be written as

\section{V.Ananthan and S.Kandasamy}




$$
(\lambda-\mu)^{4}=0
$$

Letting $y(n)=\mu^{n} x(n)$ in equation (A), we obtain

$\mu^{n+k} x(n+k)+\mu^{n+k-1}\left(a_{1}+p_{1}(n)\right)+----+$

$\mu^{n}\left(a_{k}+p_{k}(n)\right) x(n)=0$

Or

$x(n+k)+\frac{1}{\mu}\left(a_{1}+p_{1}(n)\right) x(n+k-1)+$

$\ldots \ldots \ldots+\frac{1}{\mu^{k}}\left(a_{k}+p_{k}(n)\right) x(n)=0$

The characteristic equation (15) is given by $\lambda_{k}+\frac{a_{1}}{\mu} \lambda^{k-1}+\frac{a_{2}}{\mu^{2}} \lambda^{k-2}+\cdots \ldots \ldots \ldots \ldots+\frac{a_{k}}{\mu^{k}}=0$

Which has $\mathrm{k}$-fold $\operatorname{root} \lambda=1$. If $p_{i}(n), 1 \leq i \leq k$, satisfies condition (A), then so does $\left(1 / \mu^{i}\right) p_{i}(n)$. Hence theorem (1.3) applies to (15) to yield solutions $x_{1}(n), x_{2}(n), \ldots \ldots, x_{k}(n)$ with

$x_{i}(n)=n^{i-1}(1+o(1)), \quad n \rightarrow \infty$

Consequently, there are solutions $y_{1}(n), y_{2}(n), \ldots \ldots \ldots y_{k}(n)$ of $(\mathrm{A})$ such that

$y_{i}(n)=n^{i-1}(1+o(1)) \mu^{n}$

We now summarize the above observation in the following corollary.

Corollary 2

Suppose that the polynomial (2) has a k-fold root $\mu$ and that condition (12) holds. Then

$y(n+k)+\left(a_{1}+p_{1}(n)\right) y(n+k-1)+\cdots \ldots \ldots \ldots \ldots+$

$\left(a_{k}+p_{k}(n)\right) y(n)=0$

Have k solutions $y_{1}(n), y_{2}(n), \ldots \ldots \ldots y_{k}(n)$ such that

$y_{i}(n)=n^{i-1}(1+o(1)) \mu^{n}$

(16)

Example: 1

Investigate the asymptotic behavior of solutions of the difference equation

$y(n+3)-\left(6+e^{-n-2}\right) y(n+2)+\left(12-\frac{1}{(n+1)^{4}}\right) y(n+$

\section{$1-8 y n=0$}

\section{Solution:}

The characteristic equation is given by $\lambda^{3}-6 \lambda^{2}+$ $12 \lambda-8=0$ with roots $\lambda_{1}=\lambda_{2}=\lambda_{3}=2$. Also $p_{1}(n)=-e^{-n-2}, p_{2}(n)=-1 /(n+1)^{4}$, and $p_{3}(n)=0$

All satisfy condition (12). Hence, by corollary 2 there are three solutions

$y_{1}(n)=(1+o(1)) 2^{n}$

$y_{2}(n)=n(1+o(1)) 2^{n}$, and $y_{3}(n)=n^{2}(1+o(1)) 2^{n}$.

\section{REFERENCES}

[1]. Philos, CH.G and Purnaras, I.K, 2004, The behavior of solutions of linear Volterra difference equations with infinite delay, Comput. Math. Appl. 47, 15551563.

[2]. Philos, CH.G, and Purnaras,I.K, 2005, The behavior of the solutions of periodic linear neutral delay difference equations, J. Comput. Appl. Math. 175 , 209-230.
[3]. CH. G. Philos and I. K. Purnaras, 2004, An asymptotic result for some delay difference equations with continuous variable, Advances in Difference Equations2004:1, 1-10.

[4]. Julio G. DIX, Christos G. Philos and Ioannis K. Purnaras, 2005, An asymptotic property of solutions to linear nonautonomous delay differential equations, Electron. J. Differential Equations, 10, 1-9.

[5]. CH. G. Philos and I. K. Purnaras, 2008, Sufficient conditions for the oscillation of linear difference equations with variable delay, J. Difference Equ. Appl. 14, 629-644.

[6]. Huilima, Hui Feng, Jiaofeng wang, Wandi Ding, 2014, Boundedness asymptotic behavior of positive solutions for difference equations of exponential form, NWNU-12-14.

\section{V.Ananthan and S.Kandasamy}

